\title{
La marchitez tardía del maíz (Zea mays L.) causada por Cephalosporium maydis en la Península Ibérica, y otros hongos asociados
}

\author{
Carmen Maria Ortiz-Bustos ${ }^{1}$, Ana B. García-Carneros ${ }^{1}$, Leire Molinero-Ruiz ${ }^{1}$
}

${ }^{1}$ Departamento de Protección de Cultivos. Instituto de Agricultura Sostenible- CSIC, Apdo. de Correos 4084, C.P.14080 Córdoba, España.

Autor para correspondencia: Leire Molinero-Ruiz (lmolinero@ias.csic.es)

Data de chegada: 15/04/2014. Aceito para publicação em: 23/04/2015.

$10.1590 / 0100-5405 / 1998$

\section{RESUMEN}

Ortiz-Bustos, C.M.; García-Carneros, A.B.; Molinero-Ruiz, L. La marchitez tardía del maíz (Zea mays L.) causada por Cephalosporium maydis en la Península Ibérica, y otros hongos asociados. Summa Phytopathologica, v.41, n.2, p.107-114, 2015.

Las especies de hongos de suelo asociadas a Cephalosporium maydis como agente causal de la marchitez tardía del maíz en la Península Ibérica se identificaron muestreando 19 campos con síntomas de marchitez en las principales zonas de cultivo entre 2011 y 2012. En el $47 \%$ de los campos no se identificó C. maydis, pero sí Fusarium graminearum, F. verticillioides, F. equiseti, F. proliferatum, Macrophomina phaseolina, Rhizoctonia solani y Trichoderma harzianum infectando las plantas de maíz. En los campos restantes, junto a $C$. maydis se identificaron otros hongos de suelo en porcentajes apreciables: F. verticillioides (19\%), F. proliferatum $(19 \%)$, F.equiseti (9\%), F. oxysporum (9\%) y Pythium oligandrum (9\%). E1 crecimiento vascular de C. maydis y de otras especies fúngicas en plantas de maíz se confirmó analizando plantas con marchitez procedentes de tres campos diferentes. Tanto C. maydis como F. graminearum, F. equiseti, F. proliferatum y $T$. harzianum se aislaron de la inserción entre la raíz y tallo y a $10 \mathrm{~cm}$ de altura en el tallo de las plantas. El efecto de la infección por $C$. maydis sobre la producción de las plantas de maíz se cuantificó en macetas y condiciones seminaturales en el 2011. En plantas inoculadas se obtuvo una reducción del peso de las mazorcas del $54 \%$, además de pesos de raíz y de parte aérea (tallo y hojas) significativamente menores en comparación con el control no inoculado, lo que sugiere el gran impacto económico que puede tener la marchitez tardía en condiciones naturales. Asimismo este trabajo pone de manifiesto el grado de complejidad de la etiología de la marchitez tardía, que debería ser estudiado mediante la confirmación de la patogenicidad de los hongos de suelo identificados en maíz, con el fin de determinar el papel que puede jugar cada una de estas especies en el desarrollo de la enfermedad y/o severidad de los síntomas.

Palabras claves: Etiología, Fusarium graminearum Schwabe, Fusarium proliferatum (Matsush.) Nirenberg ex Gerlach \& Nirenberg, Fusarium verticillioides (Sacc.) Nirenberg, hongos de suelo.

\section{RESUMO}

Ortiz-Bustos, C.M.; García-Carneros, A.B.; Molinero-Ruiz, L. Murcha tardia do milho (Zea mays L.) causada por Cephalosporium maydis e outros fungos associados na Península Ibérica. Summa Phytopathologica, v.41, n.2, p.107-114, 2015.

As espécies de fungos de solo associadas ao Cephalosporium maydis como agente causal da murcha tardia do milho na Península Ibérica, foram identificadas rastreando 19 campos com sintomas de murcha nas principais zonas de cultivo entre 2011 e 2012. Em 47\% dos campos não foi identificado C. maydis, mas sim Fusarium graminearum, $F$. verticillioides, F. equiseti, F. proliferatum, Macrophomina phaseolina, Rhizoctonia solani e Trichoderma harzianum que infetaram as plantas de milho. Nos restantes campos, além do C. maydis foram identificados outros fungos de solo em percentagens apreciáveis: $F$. verticillioides (19\%), F. proliferatum (19\%), F.equiseti (9\%), F. oxysporum $(9 \%) \mathrm{e}$ Pythium oligandrum (9\%). O crescimento vascular do C. maydis e de outras espécies fúngicas em plantas de milho foi confirmado através de análises de plantas com murcha procedente de três campos diferentes. Tanto C. maydis como F. graminearum, F. equiseti, F. proliferatum y $T$. harzianum foram isolados na inserção entre a raíz e o caule e a $10 \mathrm{~cm}$ de altura no caule das plantas. O efeito da infeção por C. maydis sobre a produção das plantas de milho, quantificou-se em vasos e condições semi-naturais em 2011. Em plantas inoculadas, obteve-se uma redução do peso das maçarocas de $54 \%$, além da redução dos pesos da raíz e parte aérea (folhas e caule) que foram significativamente menores em comparação com o controle não inoculado, o que sugere o grande impacto económico que pode ter o emurchecimento tardio em condições naturais. De igual forma, este trabalho realça o grau de complexidade da etiología do emurchecimento tardio, que deveria ser estudado mediante a confirmação da patogenecidade dos fungos de solo identificados no milho, com o objetivo de determinar o papel de cada uma destas espécies no desenvolvimento da doença e/ou severidade dos sintomas.

Palavras-chave: Etiología, Fusarium graminearum Schwabe, Fusarium proliferatum (Matsush.) Nirenberg ex Gerlach \& Nirenberg, Fusarium verticillioides (Sacc.) Nirenberg, fungos de solo. 


\section{ABSTRACT}

Ortiz-Bustos, C.M.; García-Carneros, A.B.; Molinero-Ruiz, L. The late wilt of corn (Zea mays L.) caused by Cephalosporium maydis and other fungi associated at the Iberian Peninsula. Summa Phytopathologica, v.41, n.2, p.107-114, 2015.

The soil-borne fungal species associated with Cephalosporium maydis, as the causal agent of late wilt of corn at the Iberian Peninsula, were identified by monitoring 19 fields showing wilt symptoms in the major crop areas between 2011 and 2012. In 47\% of the fields, C. maydis was not identified, but Fusarium graminearum, F. verticillioides, F. equiseti, F. proliferatum, Macrophomina phaseolina, Rhizoctonia solani and Trichoderma harzianum were found to infect corn plants. In the remaining fields, besides C. maydis, other soilborne fungi were identified at considerable percentages: $F$. verticillioides (19\%), F. proliferatum (19\%), F.equiseti (9\%), F. oxysporum $(9 \%)$ and Pythium oligandrum (9\%). The vascular growth of C. maydis and other fungal species in corn plants was confirmed by means of analyses with plants showing wilt from three different fields. Both C. maydis and F. graminearum, F. equiseti, F. proliferatum and T. harzianum were isolated at the insertion between the root and the stem and at $10 \mathrm{~cm}$ height on the stem of plants. The effect of infection by C. maydis on corn plant production was quantified in pots under semi-natural conditions in 2011. In inoculated plants, there was a $54 \%$ reduction in the weight of cobs, in addition to a reduction in the weight of roots and shoot (leaves and stem) which were significantly smaller compared to the non-inoculated control; this suggests the great economic impact that late wilt may have under natural conditions. Similarly, this study highlights the complexity degree of the etiology of late wilt, which should be studied by confirming the pathogenesis of the soil-borne fungi identified in the corn with the aim of determining the role of each species on the development of the disease and/or the severity of symptoms.

Additional keywords: Etiology, Fusarium graminearum Schwabe, Fusarium proliferatum (Matsush.) Nirenberg ex Gerlach \& Nirenberg, Fusarium verticillioides (Sacc.) Nirenberg, soil-borne fungi.

En la Península Ibérica se cultiva el 5.1\% del área de maíz producido en la Unión Europea (UE) y se concentra el $6.6 \%$ de la producción total. Estos porcentajes la sitúan dentro de los ocho primeros países de la UE con mayores cifras de producción y superficie en el año 2011 (4). Las condiciones climáticas predominantes en la Península Ibérica hacen que más del $90 \%$ de la superficie corresponda al cultivo de regadío, concentrándose éste mayoritariamente en los valles de los principales ríos. El maíz se cultiva en secano en Galicia en España (10) y en el Alentejo en Portugal (11).

En 2003, se detectaron por primera vez en la Península Ibérica síntomas prematuros de marchitez en algunos campos de maíz. Investigaciones realizadas en los años posteriores permitieron asociar estos síntomas con una enfermedad vascular, la marchitez tardía, causada por Cephalosporium maydis Samra, Sabet \& Hing. (12). La marchitez tardía sólo se había detectó con anterioridad en Egipto (22), India (17) y Hungría (18).

Cephalosporium maydis infecta a la planta a través de las raíces y asciende por el xilema colonizándola sistémicamente. El síntoma inicial de la enfermedad es la marchitez en hojas desde el borde hacia la nervadura central, comenzando por las hojas inferiores y ascendiendo hacia las superiores. Los síntomas aparecen en momentos próximos a la floración y van agravándose hasta poco antes de alcanzar la madurez . A medida que la marchitez de las hojas se intensifica, aparecen estrías amarillas o marrón-rojizas en los entrenudos basales del tallo. Al cabo de unos días los tallos se secan y se quiebran (12). Existen otros hongos habitantes del suelo que pueden penetrar a través de las raíces de la planta e instalarse en ella causando síntomas que suelen confundirse con los causados por C. maydis. Entre ellos cabe mencionar Fusarium moniliforme J. Sheld. (sin. F. verticillioides) y F. graminearum, causantes de pudriciones del tallo en maíz $(3,7)$. Trabajos previos han puesto de manifiesto la interacción patogénica entre $C$. maydis y otras especies fúngicas como Fusarium oxysporum Schltdl. Se ha comprobado que $C$. maydis aumenta la tolerancia a la marchitez en algodón causada por $F$. oxysporum cuando ambos hongos actúan de forma conjunta (20).

En el presente estudio se identifican las especies de hongos de suelo asociadas a C. maydis como agente causal de la marchitez tardía del maíz en la Península Ibérica, en algunos casos co-infectando las plantas. También es cuantificada la pérdida de producción debido a la infección por C. maydis en condiciones seminaturales.

\section{MATERIAL Y MÉTODOS}

Identificación de especies de hongos de suelo asociadas a $C$. maydis como agente causal de la marchitez tardía

Se realizaron prospecciones en los años 2011 y 2012 en un total de 19 campos de maíz de la Península Ibérica donde se observaron plantas que presentaban síntomas de marchitez. Los campos se localizaban en las cuencas de los ríos Guadalquivir, Guadiana, Tajo y Ebro, concretamente en las provincias de Sevilla, Badajoz, Toledo, Zaragoza, Huesca y Madrid, y en el distrito portugués de Santarem. (Tabla 1)

Las prospecciones se realizaron entre los meses de julio y octubre y las muestras consistieron en 2-6 plantas por parcela. Se tomaron muestras de la parte basal de cada planta, incluyendo $10-20 \mathrm{~cm}$ de tallo, cuello y raíces. Se desinfectaron secciones transversales de la inserción entre el tallo y la raíz con hipoclorito sódico al 20\% durante 3-4 minutos y se lavaron durante 3-4 minutos en agua destilada. Después de secarlas al aire, se sembraron en placas Petri con medio $\mathrm{CMA}_{\mathrm{S}}$ (Corn-Meal-Agar, $17 \mathrm{~g}$ de harina de maíz-agar y $0,3 \mathrm{~g}$ de sulfato de estreptomicina por litro de agua destilada). Una vez sembradas, las placas se incubaron en oscuridad a $28^{\circ} \mathrm{C}$. Transcurridas $72 \mathrm{~h}$, de las colonias fúngicas observadas en la superficie del medio de cultivo se tomaron extremos hifales que se sembraron de nuevo en $\mathrm{CMA}_{\mathrm{S}}$ para ser identificados morfológicamente. La identificación de los aislados de C. maydis se llevó a cabo a partir de las características descritas por Samra et al. (22) en medio PDA, que coinciden con las que se observan en medio CMA (12).Al procesar las muestras de plantas sintomáticas se recuperaron consistentemente otras colonias fúngicas diferentes a $C$. maydis. Extremos hifales de estas colonias se transfirieron a placas con PDA (39g de patata dextrosa-agar, $0,3 \mathrm{~g}$ de sulfato de estreptomicina por litro de agua destilada) y se incubaron en oscuridad a $25^{\circ} \mathrm{C}$ durante $72 \mathrm{~h}$. La identificación se realizó mediante observaciones de hifas, conidias y conidióforos en el microscopio y según Ainsworth et al. (1), Barnett \& Hunter (2), Leslie \& Summerell (9) y Nelson et al. (15), y se confirmó molecularmente mediante la amplificación de la región ITS del ADN ribósomico con los primers ITS-4 e ITS-5. La región amplificada se secuenció y comparó con la base de datos del NCBI utilizando la 
Tabla 1. Localización geográfica (valle, localidad y finca) de campos de maíz afectados por marchitez tardía prospectados en 2011 y 2012 , y n de plantas (muestras) analizadas en cada campo

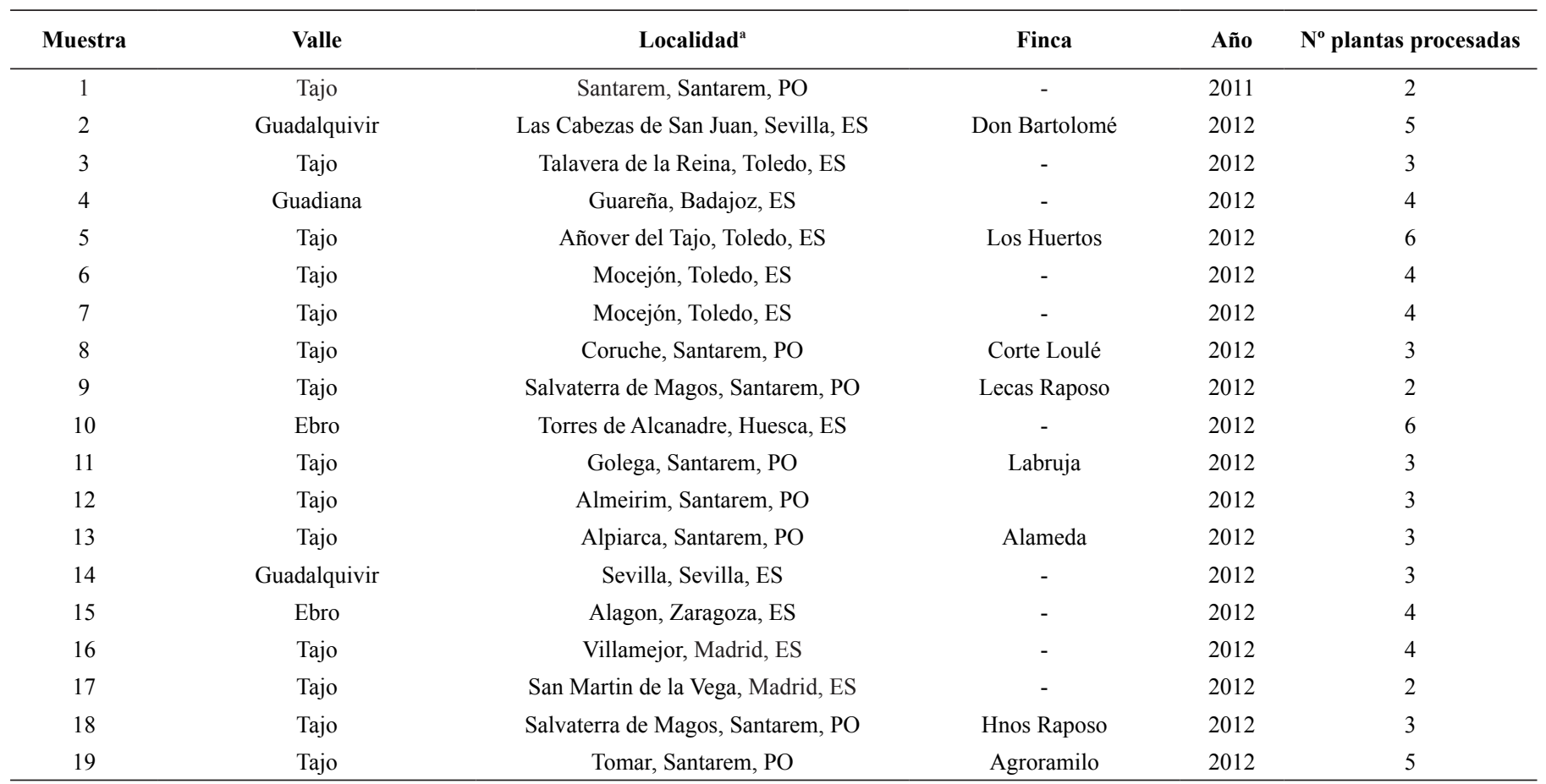

${ }^{a}$ Se indican localidad, provincia/distrito y país (ES: España y PO: Portugal) de las muestras analizadas.

$\mathrm{b}=$ nombre de la finca desconocido.

aplicación BLAST.

La co-infección vascular de C. maydis y otras especies fúngicas en plantas de maíz se estudió seleccionando tres parcelas de distinto ámbito geográfico (dos en España y una en Portugal) y tomando muestras de 2-5 plantas en cada una de ellas. (Tabla 2). En cada planta se tomaron secciones transversales de la inserción entre la raíz y el tallo, y del tallo, a una distancia de $10 \mathrm{~cm}$ de la inserción de éste con la raíz, las cuales se procesaron siguiendo el procedimiento anteriormente descrito. Las colonias de $C$. maydis se identificaron morfológicamente y las restantes especies fúngicas se identificaron tanto morfológicamente como molecularmente según las metodologías descritas previamente.

Una vez identificadas, todas las colonias fúngicas, incluidas las de C. maydis, se conservaron en la colección mantenida en el Instituto de Agricultura Sostenible, CSIC, Córdoba (España).

Efecto de la infección de $C$. maydis sobre la producción de maíz en condiciones seminaturales

Para determinar el efecto de la enfermedad en la producción de maíz se realizó un experimento en condiciones seminaturales (umbráculo) entre los meses de marzo y julio del año 2011 en Córdoba. Se eligió un aislado de $C$. maydis seleccionado en prospecciones anteriores realizadas en el año 2009. Se inocularon 6 plantas (repeticiones) de la variedad susceptible de maíz V1 (Monsanto Agricultura España, S.L.). Como control se utilizaron 6 plantas no inoculadas de la misma variedad.

La inoculación de las plantas se realizó mediante la adición de 200 gramos de granos de trigo colonizados por $C$. maydis a un sustrato estéril ( 1 arena: 1 limo, v/v) en macetas de 5 litros, donde se sembraban las semillas de maíz previamente germinadas y con una longitud de raíz de 5-10 mm. En el caso de las plantas control, se emplearon granos de trigo sin el hongo.

El trigo se esterilizó en autoclave, se infestó con discos $(9 \mathrm{~mm}$ de diámetro) de 10 días de edad de C. maydis en PDA (8 discos por cada $250 \mathrm{~g}$ de trigo). Los frascos se incubaron a temperatura ambiente durante 4 semanas.

Las plantas se regaron a demanda durante el experimento, se abonaron con $2.3 \mathrm{~g}$ de urea por maceta tres veces durante el primer mes tras la siembra y con una solución nutritiva completa (6) dos veces por semana desde la siembra hasta el levantamiento del experimento.

Transcurridas 15 semanas de la inoculación, se evaluaron los

Tabla 2. Procedencia de plantas de maíz con síntomas de marchitez tardía seleccionadas para la identificación de la co-infección de Cephalosporium maydis y otras especies fúngicas en ellas

\begin{tabular}{|c|c|c|c|}
\hline \multicolumn{3}{|c|}{ Origen geográfico } & \multirow{2}{*}{$\mathbf{N}^{0}$ plantas sintomáticas muestreadas } \\
\hline Valle & Localidad & Finca & \\
\hline Tajo & Tomar, Santarem, PO & Agroramilo & 5 \\
\hline Guadalquivir & Posadas, Córdoba, ES & Mingahover & 3 \\
\hline Guadalquivir & Posadas, Córdoba, ES & La Estrella & 2 \\
\hline
\end{tabular}


síntomas aéreos de las plantas siguiendo una escala del 1 al 9 siendo 1 el valor correspondiente a una planta sana y 9 a una planta con toda la parte aérea marchita. También se observaron los síntomas en las raíces asignando a cada planta un valor de 0 a 4 donde el valor 0 representa a raíces con aspecto normal y el valor 4 a raíces completamente necrosadas. Además se pesaron la raíz y la parte aérea (tallo, hojas y mazorcas) de cada planta.

El diseño experimental usado fue completamente aleatorizado. Los datos de los síntomas finales en la raíz (previa transformación sqrt [(valor escala +0.5$)]$ ) y en la parte aérea, y los pesos finales de ambas partes de la planta se procesaron estadísticamente mediante un análisis de varianza (ANOVA). Cuando existían diferencias significativas se aplicó el test de mínima diferencia significativa protegida de Fisher al nivel de probabilidad de $5 \%$.

\section{RESULTADOS}

Identificación de especies de hongos de suelo asociadas a $C$. maydis como agente causal de la marchitez tardía

Se aisló C. maydis en el 53\% de los campos prospectados (Tabla 3), encontrándose éstos localizados en los valles de los ríos Guadalquivir, Guadiana y Tajo. Por otro lado, C. maydis se identificó en los dos años de muestreo y tanto en España como en Portugal. Todos los aislados mostraron la morfología descrita por Samra et al. (22) y su patogenicidad se ha confirmado en trabajos previos $(5,16)$.

La frecuencia con que se identificaron otras especies fúngicas en los mismos o en diferentes campos de maíz en los que se identificó $C$. maydis se muestra en la Tabla 3.

Tabla 3. Frecuencia (\%) de identificación de C. maydis y otras especies fúngicas en los 19 campos de maíz prospectados en la Península Ibérica en los años 2011 y 2012

\begin{tabular}{lcc}
\hline Hongos identificados & Total de campos & $\mathbf{\%}$ \\
\hline Sólo otras especies fúngicas & 9 & 47 \\
C. maydis y otras especies fúngicas & 10 & 53 \\
\hline
\end{tabular}

Todos los análisis moleculares dieron valores de similitud del 92$100 \%$ entre la secuencia de ADN y las secuencias depositadas en la base de datos de GenBank. (Tabla 4)

En 9 de las 19 prospecciones efectuadas no se consiguió aislar $C$. maydis en plantas con síntomas de marchitez tardía, sin embargo fue posible recuperar otros hongos. En la Figura 1 se muestran las especies fúngicas halladas y el porcentaje de aparición de las mismas. Como se muestra en la Figura 1A, el género que se aisló con mayor frecuencia en las plantas de estas parcelas (53\%) fue Fusarium Link. si bien el $5 \%$ de los aislados de este género no se pudieron identificar a nivel de especie. Las especies infectivas de maíz más representativas en estos campos fueron $F$. graminearum y $F$. verticillioides que aparecieron, en ambos casos, en un $16 \%$ de los aislamientos, seguidas por Fusarium equiseti (Corda) Sacc., Trichoderma harzianum Rifai y Macrophomina phaseolina (Tassi) Goid., ambas en un $11 \%$. Otros hongos de suelo importantes que se identificaron infectando maíz en estos campos fueron $F$. proliferatum y Rhizoctonia solani J.G. Kühn.

Paralelamente, en los campos prospectados donde se identificó C. maydis, se aislaron otras especies fúngicas que infectaban las plantas de maíz (Tabla 3). La frecuencia de aparición de estos hongos en los aislamientos se muestra en la Figura 1B. Además del género
Tabla 4. Especies fúngicas identificadas y porcentaje (\%) de similitud entre la secuencia analizada y las secuencias almacenadas en la base de datos de GenBank

\begin{tabular}{|c|c|c|}
\hline Muestra & $\begin{array}{l}\text { Especies fúngicas } \\
\text { identificada }^{\mathrm{a}}\end{array}$ & $\begin{array}{c}\text { Referencia GenBank } \\
\text { (\% Similitud) }\end{array}$ \\
\hline \multirow[t]{2}{*}{1} & Cephalosporium maydis & $--^{b}$ \\
\hline & Fusarium proliferatum & EU821492.1 (100) \\
\hline 2 & Rhizoctonia solani & --- \\
\hline 3 & Fusarium sp. & --- \\
\hline \multirow[t]{3}{*}{4} & F. proliferatum & EU821492.1 (100) \\
\hline & Gibberella moniliformis & HQ176445.1 (100) \\
\hline & Pythium acanthophoron & AB355644.1 (99) \\
\hline \multirow[t]{5}{*}{5} & C. maydis & --- \\
\hline & Fusarium equiseti & GQ505694.1 (100) \\
\hline & G. moniliformis & HQ176445.1 (100) \\
\hline & Gibberella sacchari & AF455450.1 (100) \\
\hline & Phaeocytostroma ambiguum & FR748043.1 (100) \\
\hline \multirow[t]{3}{*}{6} & C. maydis & --- \\
\hline & G. moniliformis & EU364864.1(100) \\
\hline & Pythium oligandrum & HQ832778.1 (100) \\
\hline \multirow[t]{2}{*}{7} & C. maydis & --- \\
\hline & G. moniliformis & HQ176445.1 (100) \\
\hline \multirow[t]{2}{*}{8} & Coprinopsis urticicola & HQ847015.1 (92) \\
\hline & Hypocrea lixii & JQ040357 (100) \\
\hline \multirow[t]{3}{*}{9} & C. maydis & --- \\
\hline & F. equiseti & EU326202.1 (100) \\
\hline & P. oligandrum & HQ832778.1 (99) \\
\hline \multirow[t]{2}{*}{10} & Gibberella zeae & HQ832817.1 (100) \\
\hline & Stenocarpella maydis & FR748057.1 (99) \\
\hline \multirow[t]{2}{*}{11} & C. maydis & --- \\
\hline & F. proliferatum & EU821492.1 (100) \\
\hline \multirow[t]{2}{*}{12} & F. equiseti & JQ936260.1 (99) \\
\hline & G. moniliformis & HQ176445.1 (100) \\
\hline \multirow[t]{4}{*}{13} & C. maydis & --- \\
\hline & Ceratobasidium sp. & HM623632.1 (92) \\
\hline & Fusarium oxysporum & JF731234.1 (100) \\
\hline & R. solani & JQ946295.1 (100) \\
\hline 14 & Macrophomina phaseolina & GU046905.1 (99) \\
\hline \multirow[t]{3}{*}{15} & F. equiseti & AY147362.1 (100) \\
\hline & G. zeae & --- \\
\hline & M. phaseolina & GU046905.1 (99) \\
\hline \multirow[t]{3}{*}{16} & C. maydis & --- \\
\hline & Gibberella intermedia & JX241655.1 (99) \\
\hline & H. lixii & --- \\
\hline \multirow[t]{4}{*}{17} & G. moniliformis & --- \\
\hline & G. zeae & --- \\
\hline & H. lixii & --- \\
\hline & Pythium periplocum & GU811234.1 (99) \\
\hline \multirow[t]{4}{*}{18} & C. maydis & --- \\
\hline & F. oxysporum & KC254035.1 (99) \\
\hline & Gibberella fujikuroi & HM776427.1 (100) \\
\hline & G. zeae & --- \\
\hline \multirow[t]{3}{*}{19} & C. maydis & --- \\
\hline & F. proliferatum & --- \\
\hline & G. moniliformis & JF499676.1 (99) \\
\hline
\end{tabular}

${ }^{\text {a }}$ Se presenta el anamorfo o el teleomorfo de cada especie según la referencia de GenBank.

b --- = la especie fúngica se identificó morfológicamente. 
Ceratobasidium D.P. Rogers se aislaron 11 especies diferentes, perteneciendo 7 de ellas al género Fusarium, que representó el 71 $\%$ del total de especies fúngicas aisladas. Las especies fúngicas más frecuentemente aisladas de las plantas junto con $C$. maydis fueron $F$. proliferatum y $F$. verticillioides, constituyendo un $19 \%$ en ambos casos. El resto de hongos recuperados correspondieron a las especies Pythium oligandrum Drechsler, $R$. solani, T. harzianum y Phaeocytostroma ambiguum (Mont.) Petr.

En la Figura 2 se muestran de forma esquemática las especies fúngicas aisladas en el tallo y en la inserción raíz-tallo en plantas enfermas procedentes de las tres fincas evaluadas. La frecuencia de aislamiento de cada una de las especies se expresa, en cada finca, como número de plantas donde se ha aislado la especie respecto al total de plantas analizadas.
Cephalosporium maydis fue recuperado en todas las plantas analizadas en las fincas cordobesas y en 2 de las 5 plantas procedentes de la finca de origen portugués. En plantas de las tres fincas fue posible aislar C. maydis en el tallo. Sin embargo, del material vegetal seccionado de la inserción raíz-tallo, sólo se recuperó C. maydis en las plantas procedentes de las fincas de Córdoba.

Junto con C. maydis, en las plantas sintomáticas se hallaron otras 5 especies fúngicas distintas pertenecientes a los géneros Fusarium y Trichoderma Pers. De ellas, sólo F. proliferatum fue aislada en plantas de fincas distintas.

En la finca Agroramilo únicamente se identificaron especies de Fusarium en co-infección con C. maydis. Sólo $F$. equiseti y $F$. proliferatum se recuperaron de las dos zonas analizadas de las plantas sintomáticas. Las otras especies infectivas de maíz halladas en esta
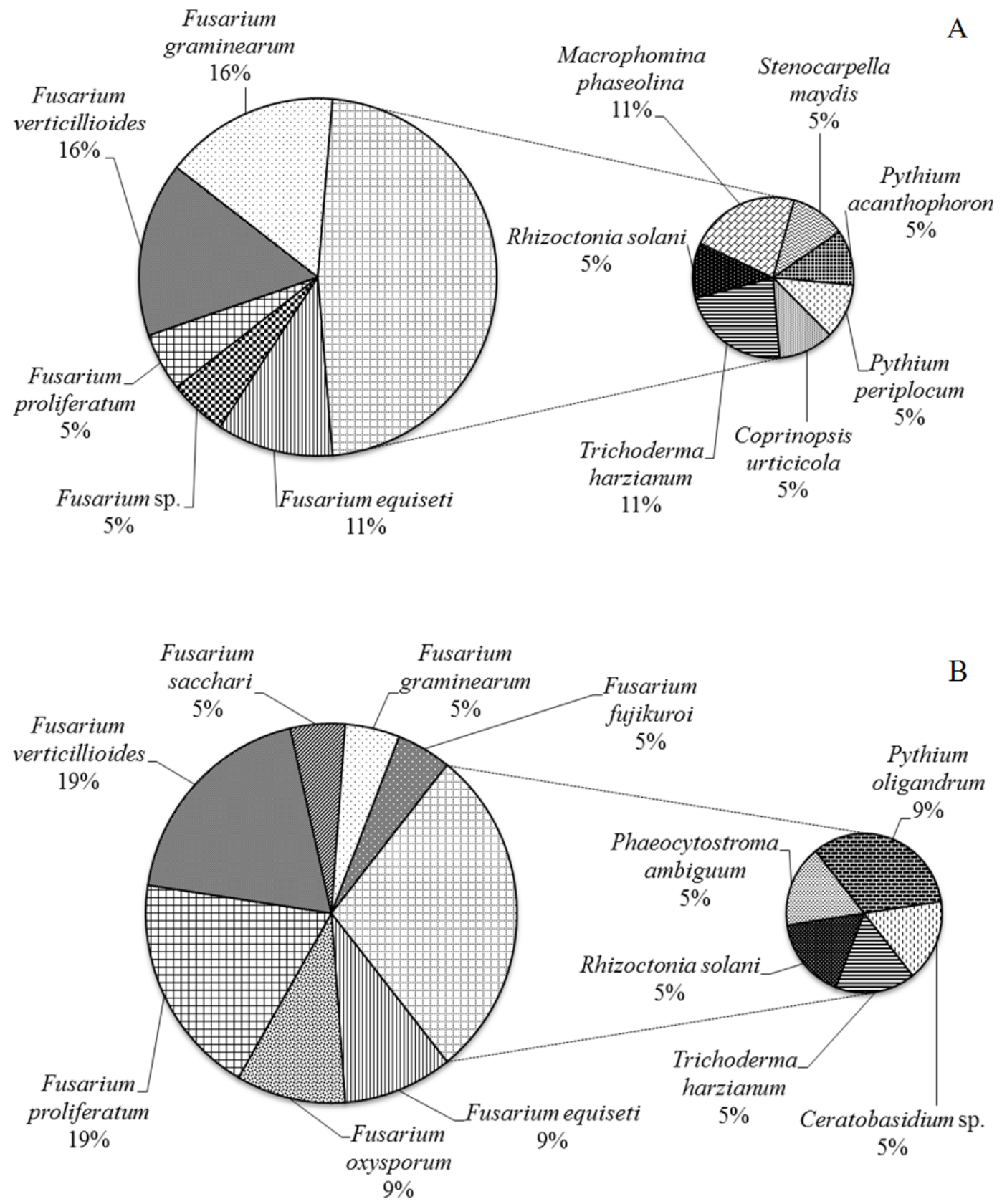

Figura 1. Frecuencia (\%) de identificación de distintas especies fúngicas en los aislamientos realizados a partir de plantas de maíz con síntomas de marchitez tardía en las que no se detectó a Cephalosporium maydis (A) y en las que si se recuperó C. maydis (B). 
finca, $F$. graminearum y $F$. verticillioides, aparecieron exclusivamente en el tallo y en la inserción de éste con la raíz respectivamente.

En cada una de las dos fincas cordobesas (Mingahover y La Estrella), se detectó sólo un hongo distinto a C. maydis y en una sola planta del total de plantas analizadas en cada una. De este modo, se aisló F. proliferatum en la finca Mingahover y T. harzianum en La Estrella.

Por último, y respecto a la distribución de especies en las plantas, tanto $F$. equiseti como $F$. proliferatum se aislaron en las dos partes analizadas (finca Agroramilo). En la inserción de la raíz con el tallo pero no más arriba en la planta se recuperaron $F$. verticillioides (finca Agroramilo) y $F$. proliferatum (finca Mingahover). Por el contrario, los hongos $F$. graminearum (finca Agroramilo) y T. harzianum (finca La Estrella) se aislaron del tallo pero no de su inserción con la raíz.

Efecto de la infección de $C$. maydis sobre la producción de maíz en condiciones seminaturales

El análisis estadístico ANOVA estableció diferencias significativas entre las plantas inoculadas y las plantas control tanto de los síntomas aéreos como de los de raíz ( $\mathrm{p}<0.0001$ en ambos casos). Al comparar los valores medios de ambas variables de la enfermedad se observó que, mientras las plantas inoculadas alcanzaron valores de 8.5 y 3.5 en la escala de síntomas aéreos y de raíz respectivamente, las no inoculadas tuvieron los valores mínimos de ambas escalas, es decir, 1 en la escala de síntomas aéreos y 0 en la de raíz (Figura 3A).

Respecto al análisis entre los pesos de las plantas inoculadas y no inoculadas, el ANOVA arrojó diferencias significativas en peso de parte aérea (tallo más hojas) $(\mathrm{p}<0.0001)$, peso de raíz $(\mathrm{p}=0.0002)$ y peso de mazorca $(\mathrm{p}=0.0001)$. Las plantasinoculadas redujeron el peso de su parte aérea en un $77 \%$ respecto a las plantas control y el peso de la raíz de las plantas inoculadas disminuyó en un $83 \%$ respecto al de las plantas sin inocular. En cuanto a la producción, se detectó un efecto similar al descrito anteriormente: Las plantas inoculadas experimentaron una reducción del peso de mazorca del 54\% respecto a las plantas control (Figura 3B).

\section{DISCUSION}

La presencia de C. maydis en los valles de los ríos Tajo, Guadiana y Guadalquivir ha sido referida recientemente (16), pero por el momento los síntomas de marchitez observados en las muestras procedentes del valle del río Ebro no se han podido asociar a infecciones por el mismo hongo. Este hecho podría estar relacionado con las diferentes condiciones medioambientales de la zona en comparación con las del centro-sur de la Península. De hecho, la climatología del centro-sur, con menor disponibilidad de agua y elevada temperatura, es más parecida a la referida por otros autores como favorable para la infección del maíz en Egipto e India (23). No obstante, la ausencia de infecciones por $C$. maydis en el valle del Ebro debería ser investigada en futuros trabajos.

Respecto a la identificación en algunas plantas con síntomas de F. graminearum y F. verticillioides pero no de C. maydis, no resulta inesperado, ya que estos hongos de suelo también son patógenos de maíz y los síntomas que originan en las plantas son similares $(8,24)$. Otras especies identificadas en las muestras, en ausencia de C. maydis, fueron

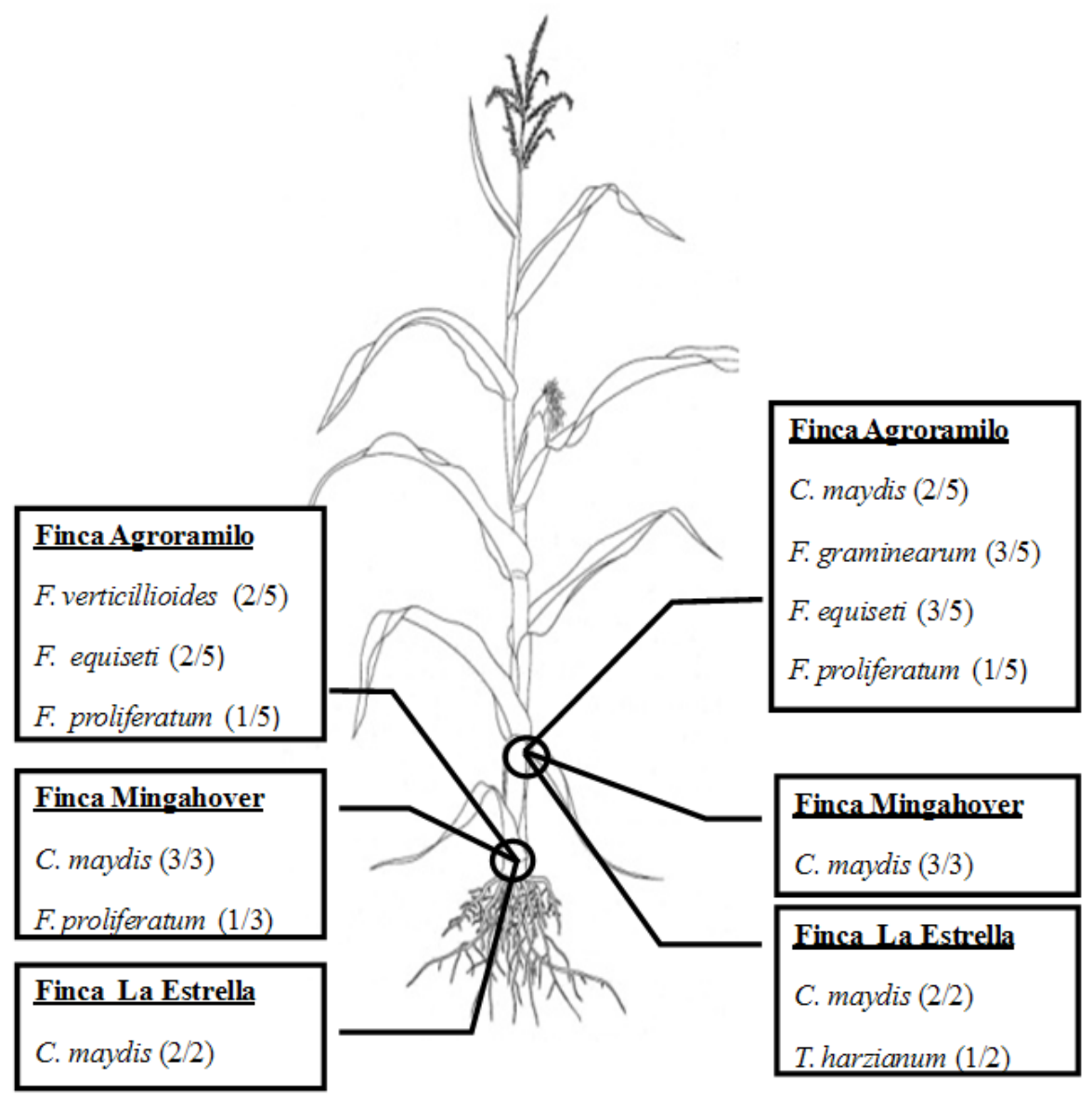

Figura 2. Esquema de una planta de maíz con la distribución de especies fúngicas aisladas en el tallo y en la inserción raíz-tallo; y frecuencia de aislamiento de cada una de las especies expresada como número de plantas donde se ha aislado la especie respecto al total de plantas analizadas en cada finca. 

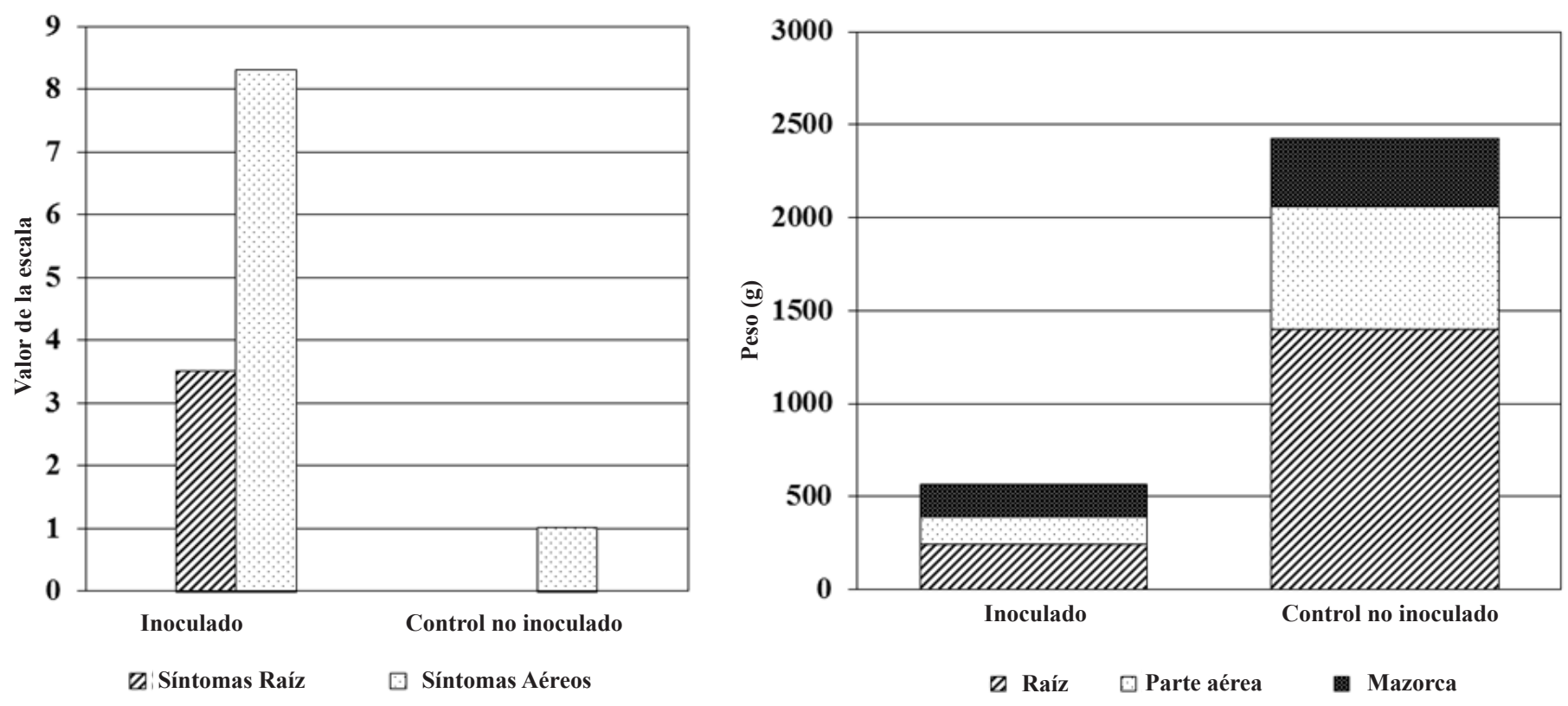

Figura 3. Valores medios de síntomas aéreos y en raíces (A) y de los pesos de raíces, parte aérea (hojas y tallos) y mazorcas (B) de plantas de maíz inoculadas con C. maydis en comparación con plantas control no inoculadas.

F. proliferatum, M. phaseolina y Stenocarpella maydis (Berk.) B. Sutton, hongos de suelo que también son patógenos de maíz y cuya presencia en las plantas está asociada a pudriciones de tallo $(13,19,24)$. No obstante, la patogenicidad de los aislados de M. phaseolina y $S$. maydis, pero, sobre todo, de los de las diferentes especies de Fusarium identificadas en este trabajo, debería confirmarse en futuros experimentos ad hoc. Por último, otros hongos de suelo más frecuentemente identificados junto con $C$. maydis fueron $F$. verticillioides y $F$. proliferatum. También se identificaron $F$. graminearum, $R$. solani, F. equiseti y $T$. harzianum. Excepto $T$. harzianum, que por otro lado tiene una amplia capacidad colonizadora (14) todas las demás especies pueden infectar maíz y en algunos casos se han observado junto con $C$. maydis al realizar aislamientos de tejidos $(19,21)$. Los resultados del análisis en distintas partes de plantas de maíz enfermas confirmaron el comportamiento vascular de $C$. maydis, pero también permitieron identificar otros hongos tanto en la raíz como en el tallo de las plantas. Estos resultados sugieren que la marchitez tardía puede estar asociada a la presencia de más de un agente fúngico infectivo. Así, la enfermedad podría tener una etiología compleja, pues la infección y el establecimiento de $C$. maydis en las plantas pueden estar acompañados de la penetración de especies como $F$. proliferatum o $F$. graminearum. Estos hongos podrían actuar como patógenos per se, o ser meros oportunistas que se instalan en la planta tras la penetración previa por $C$. maydis. En cualquier caso, resulta de interés la investigación de la patogenicidad de las especies identificadas en este trabajo, especialmente $F$. proliferatum o F. graminearum, en combinación con C. maydis, con el fin de determinar qué papel puede jugar cada una de ellas en el desarrollo de la enfermedad en las plantas de maíz.

En cuanto al efecto de C. maydis sobre la producción de maíz, nuestros resultados, obtenidos en condiciones seminaturales de umbráculo y crecimiento de las plantas en maceta, mostraron que las plantas inoculadas tuvieron una reducción del peso de las mazorcas del $54 \%$, además de pesos de raíz y de parte aérea (tallo y hojas) significativamente menores. Estos resultados guardan similitud con los obtenidos en trabajos previos (16) y muestran el elevado impacto económico que puede tener la marchitez tardía del maíz en el campo.

\section{AGRADECIMIENTOS}

Trabajo parcialmente financiado por el proyecto PIE2009401120 (CSIC, MICINN). Los autores agradecen a las empresas Pioneer HiBred Agro Servicios España, S.L. y Monsanto Agricultura España, S.L. la cesión de semillas de maíz así como la colaboración en la obtención de algunas de las muestras analizadas. Los autores también expresan su agradecimiento a S. Benquerenca por la traducción del resumen al portugués.

\section{REFERENCIAS BIBLIOGRAFICAS}

1. Ainsworth, G.C. Introduction and keys to higher taxa. In: Ainsworth, G.C.; Sparrow, F.K.; Sussman, A.S. The Fungi: an advanced treatise. Nueva York: Academic Press, 1973.v. IV A., p.1-7.

2. Barnett, H.L.; Hunter, B.B. Illustrated genera of imperfect fungi. Saint Paul: American Phytopathological Society Press,1998. 218p.

3. Christensen, J.J.; Wilcoxson, R.D. Stalk rot of corn. Saint Paul: American Phytopathological Society Press, 1966. 59p (Monograph,3).

4. FAOSTAT. Maize harvested areas and production quantity in 2011.Rome: FAO, 2013. Available at: <http://faostat.fao.org/> Accessed on: 11 Mar. 2013.

5. García-Carneros, A.B.; Giron, I.; Molinero-Ruiz, L. Aggressiveness of Cephalosporium maydis causing late wilt of maize in Spain. Communications in agricultural and applied biological sciences, Gent, v.77, n.3, p.173-179, 2012 .

6. Hoagland, D.R.; Arnon, D.I. The water culture method for growing plants without soil. Berkeley: California Agricultural Experiment Station, 1950. (Circ.,347).

7. Kucharek, T.A.; Kommedalh, T. Kernel infection and corn stalk rot caused by Fusarium moniliforme. Phytopathology, Saint Paul, v.56, p.983-984, 1966. 
8. Leslie, J.F.; Pearson, C.A.S.; Nelson, P.E.; Toussoun, T.A. Fusarium species from corn, sorghum, and soybean fields in the central and eastern United States. Phytopathology, Saint Paul, v.80, p.343-350, 1990.

9. Leslie, J. F.; Summerell, B. A. The Fusarium Laboratory Manual. Ames: Blackwell Publishing Professional, 2006. 387p.

10.España. Ministerio de Agricultura, Alimentación y Medio Ambiente. Anuario de Estadística 2011. Ministerio de Agricultura, Alimentación y Medio Ambiente. Madrid. Available at: < http://www.magrama.gob. es/es/> Accessed on: 10 Mar. 2013.

11.Portugal. Ministerio da Agricultura, do Desenvolvimiento Rural e Das Pescas MADRP. Cultura Arvenses. Diagnóstico Sectorial. Gabinete de Planeamiento e Políticas. Lisboa. 2007. Available at:< http://www. gpp.pt/> Accessed on: 3 Mar.2013.

12. Molinero-Ruiz, M.L.; Melero-Vara, J.M.; Mateos, A. 2010. Cephalosporium maydis, the Cause of Late Wilt in Maize, a Pathogen New to Portugal and Spain. Plant Disease, Saint Paul, v.94, n.3, p.379, 2010 .

13. Molinero-Ruiz, L.; Rubio-Pérez, E.; González-Domínguez, E.; Basallote-Ureba, M.J. Alternative hosts for Fusarium spp. causing crown and root rot of asparagus in Spain. Journal of Phytopathology, Göttingen, v.159, n.2, p.114-116, 2011.

14. Mukherjee, P.K.; Horwitz, B.A.; Herrera-Estrella, A.; Schmoll, M., Kenerley, C.M. Trichoderma Research in the Genome Era. Annual Review of Phytopathology, Palo Alto, v.51, p.105-129, 2013.

15. Nelson, P.E.; Toussoun, T.; Marasas, W. Fusarium species: an illustrated manual for identification. Stage College: Pennsylvania State University Press, 1983. 193p.

16. Ortiz-Bustos, C.M.; Testi, L.; García-Carneros, A.B.; Molinero-Ruiz,
L. Geographic distribution and virulence of Harpophora maydis in the Iberian Peninsula, and thermal detection of maize late wilt. (under review)

17. Payak, M.M.; Lal, S.; Lilaramani, J.; Renfro, B.L. Cephalosporium maydis a new threat to maize in India. Indian Phytopathology, New Delhi, v.23, p.562-569, 1970.

18. Pecsi, S.; Nemeth, L. Appearance of Cephalosporium maydis Samra, Sabet and Hingorani in Hungary. Noticias. Faculteit Landbouwkundige en Toegepaste Biologische Wetenschappen Universiteit Gent, Gent, v.63, p.873-877, 1998.

19. Programa de Maíz del CIMMYT. Enfermedades del Maíz: una guía para su identificación en el campo. 4. ed., Cimmyt, 2004.119p.

20. Sabet, K.A.; Samra, A.S.; Mansour, I.S. Interaction between Fusarium oxysporum, F. vasinfectum, and Cephalosporium maydis on cotton and maize. Annals of Applied Biology, London, v.58, p.93-101,1966.

21. Saleh, A.A.; Zeller, K.A.; Ismael, A.M.; Fahmy, Z.M.; El-Assiuty, E.M.; Leslie, J.F. Amplified fragment length polymorphism diversity in Cephalosporium maydis from Egypt. Phytopathology, Saint Paul, v.93, p.853-859, 2003.

22. Samra, A.S.; Sabet, K.A.; Hingorani, M.K. Late wilt disease of maize caused by Cephalosporium maydis. Phytopathology, Saint Paul, v.53, p.402-406, 1963 .

23. Singh, S.D.; Siradhana, B.S. Influence of some environmental conditions on the development of late wilt of maize induced by Cephalosporium maydis. Indian Journal of Mycology and Plant Pathology, Udaipur, v.17, p.1-5, 1987.

24. White, D.G. Compendium of corn diseases. 3rd ed. Saint Paul: American Phytopathological Society Press, 1999. 78p. 